\title{
Importance of Time to Chemotherapy Initiation in Small Cell Lung Cancer
}

\section{Gregory J Kubicek ${ }^{1 *}$, Rachel Koehler ${ }^{2}$, Matthew J Rossi ${ }^{2}$, Christian Squillante $^{3}$, Alexander Hageboutros ${ }^{3}$, Melvin Pratter ${ }^{4}$, Stephen Akers ${ }^{4}$ and Polina Khrizman ${ }^{3}$}

${ }^{1}$ MD Anderson-Cooper Cancer Center, Radiation Oncology Camden NJ, USA

${ }^{2}$ Cooper Medical School, Rowan University, Camden NJ, USA

${ }^{3} \mathrm{MD}$ Anderson-Cooper Cancer Center, Medical Oncology Camden NJ, USA

${ }^{4} \mathrm{MD}$ Anderson-Cooper Cancer Center, Pulmonary Medicine, Camden NJ, USA

*Corresponding author: Gregory J Kubicek, Department of Radiation Oncology, Cooper University Hospital, One Cooper Plaza, Camden NJ, 08103, USA, Tel: 856-342-2300, Fax: 856-365-8504

\begin{abstract}
Background: Small cell lung cancer (SCLC) is an aggressive malignancy with a short median survival time. Because of the rapid growth rate there may be an advantage to emergently beginning chemotherapy as soon as SCLC diagnosis is made.

Methods: All SCLC patients evaluated at Cooper University Hospital from January 2011 to September 2014 were reviewed. Multiple clinical factors were analyzed including timing between diagnosis and start of chemotherapy.

Results: A total of 75 patients were analyzed. On univariate analysis there was a survival detriment to early initiation of chemotherapy. With multivariate analysis the difference in survival disappeared. With logistic regression, the only variable that was related to overall survival was stage (extensive versus limited). We did not find any subset that benefited from early initiation of chemotherapy.

Conclusions: Mortality and cumulative survival time were not improved by early initiation of chemotherapy for any patient subset. Only stage at diagnosis was predictive for mortality and cumulative survival. Our data appears to show that urgency in starting chemotherapy has little bearing on survival in patients diagnosed with SCLC. The data suggest that there is no detriment to a non-urgent start time for chemotherapy.
\end{abstract}

\section{Introduction}

Small Cell Lung Cancer (SCLC) is aparticularly aggressive lung malignancy characterized by a rapid doubling time, symptomatic disease, and early development of metastatic disease $[1,2]$. The cellular proliferation rate is much higher in comparison to other types of lung cancer, which has contributed tothe view of newly diagnosed SCLC as an "oncologic emergency". Some oncologists will urge the start of chemotherapy as soon as possible (sometimes within 24 hours of diagnosis) given the potential for aggressive growth and metastasis. Similarly, some institutions will keep a patient with newly diagnosed lung cancer admitted to the hospital until final pathology is available so that in the event that the final diagnosis is SCLC chemotherapy can be initiated immediately as an in-patient.

The theoretical rationale for emergent start of chemotherapy is based on the perceived rapid growth rate for SCLC. Since the tumor has such rapid growth potential, earlier chemotherapy start time may allow for an increase in cure rate for limited stage patients and provide improved disease control in extensive stage patients. While there may be a theoretical benefit to early initiation of chemotherapy, there is also a downside. Emergent initiation of therapy may overwhelm patients who haven't had time to properly process their disease status (including potentially compromising the integrity of informed consent), strain hospital systems with the costs of inpatient chemotherapy and delayed discharge, and interfere with proper disease staging.

Citation: Kubicek GJ, Koehler R, Rossi MJ, Squillante C, Hageboutros A, et al. (2019) Importance of Time to Chemotherapy Initiation in Small Cell Lung Cancer. Int J Cancer Clin Res 6:111. doi.org/10.23937/23783419/1410111

Accepted: April 18, 2019; Published: April 20, 2019

Copyright: (c) 2019 Kubicek GJ, et al. This is an open-access article distributed under the terms of the Creative Commons Attribution License, which permits unrestricted use, distribution, and reproduction in any medium, provided the original author and source are credited. 
Given that little data exists to evaluate the efficacy of emergent chemotherapy in patients with SCLC, this study aims to determine if overall survival differs between patients based on time for initiation of chemotherapy.

\section{Methods}

The data acquired in this study came from a retrospective examination of SCLC patients at one institution. 102 SCLC patients were identified from a tumor registry. Twenty seven patients were removed due to missing data in the electronic health record. Multiple clinical and demographic data were collected including performance status, time from diagnosis to start of chemotherapy, reported time of presenting symptoms, staging at diagnosis, co-morbidities, age at diagnosis, type and number of cycles of chemotherapy, radiation treatment, smoking history, and reported

Table 1: Cohort Demographics.

\begin{tabular}{|c|c|}
\hline Variable & Number \\
\hline \multicolumn{2}{|l|}{ Gender } \\
\hline Male & 37 \\
\hline Female & 38 \\
\hline \multicolumn{2}{|l|}{ Age at Diagnosis (Rounded Down) } \\
\hline Median in Years & 65 \\
\hline Range in Years & $49-87$ \\
\hline \multicolumn{2}{|l|}{ KPS } \\
\hline$>70$ & $84.6 \%(33 / 39)$ \\
\hline$<70$ & $15.4 \%(6 / 39)$ \\
\hline \multicolumn{2}{|l|}{ Time from symptom to diagnosis } \\
\hline Median in Days & 21 \\
\hline Range in Days & $0-180$ \\
\hline \multicolumn{2}{|l|}{ Chemotherapy cycles } \\
\hline Median & 6 \\
\hline Range & $0-14$ \\
\hline \multicolumn{2}{|l|}{ Co-morbidities } \\
\hline Median & 2 \\
\hline Range & $0-5$ \\
\hline Patients with any comorbidity & $88 \%(61 / 69)$ \\
\hline \multicolumn{2}{|l|}{ Radiation } \\
\hline Yes & $65.33 \%(49 / 75)$ \\
\hline No & $34.66 \%(26 / 75)$ \\
\hline \multicolumn{2}{|l|}{ Smoking history } \\
\hline Yes & 69 \\
\hline No & 1 \\
\hline \multicolumn{2}{|l|}{ Pack years } \\
\hline Median & 40 \\
\hline Range & $0-112$ \\
\hline \multicolumn{2}{|l|}{ Staging } \\
\hline Limited & 26 \\
\hline Extensive & 47 \\
\hline
\end{tabular}

number of pack-years. Time from presentation was defined as the time of first reported symptoms until time of actual diagnosis.

The median age was 65. 22 patients had limited disease while 47 had extensive disease (Table 1). The median time from diagnosis to starting chemotherapy was 23 days (range 3 to 63 days). The types of chemotherapy used (first line chemotherapy options) included carboplatin, cisplatin, etoposide, topotecan, and paclitaxel.

Documented Karnofsky Performance Status (KPS) and Eastern Cooperative Oncology Group (ECOG) scores were used to evaluate a patient's performance status depending on the physical and their preference. The ECOG scores were converted to a KPS score based off of a scale for efficient statistical evaluation. Comorbidities (coronary artery disease, history of $\mathrm{Ml}$, hypertension, hyperlipidemia, peripheral artery disease, CHF, diabetes, hepatitis, COPD, history of PE, history of malignancy, tuberculosis) were assessed and patients were stratified into groups with less than or greater than 3 total comorbidities. Only the first round of chemotherapy was analyzed in this study (chemotherapy timing with second line chemotherapy after progression was not examined).

\section{Results}

Median time from diagnosis to last follow up or death is 311 days (Range 7-1274 days). At last follow up 22 of the 75 patients (29\%) were still alive. The median follow-up time for the surviving group is 548 days.

On univariate analysis, there was an improvement in overall survival in patients with late ( $>14$ days) delay between diagnosis and chemotherapy initiation. Kaplan Meier curves demonstrate a significant difference in the time of survival between the groups that received chemotherapy less than 14 days from diagnosis compared to those that received chemo greater than 14 days from diagnosis $(p=0.008)$. Median survival for patients started chemotherapy $>14$ days was 363 days compared to 286.5 days for starting chemotherapy $<14$ days (Figure 1). Examining extensive and limited stage patients separatelythere is a trend for improved survival for limited stage patients who started chemotherapy $>$ 14 days from diagnosis. There was no relation between survival for extensive stage patients based on timing of chemotherapy initiation. When time of survival was examined with time from diagnosis to chemotherapy initiation as a continuous variable there was a trend towards improved outcome with later chemotherapy initiation (Pearson coefficient of 0.22 with a $p$-value of 0.058).

On multivariate analysis chemotherapy start time did not remain significant. Using a Cox proportional hazard model we analyzed age at diagnosis, performance status, comorbidities, stage (limited versus extensive), 


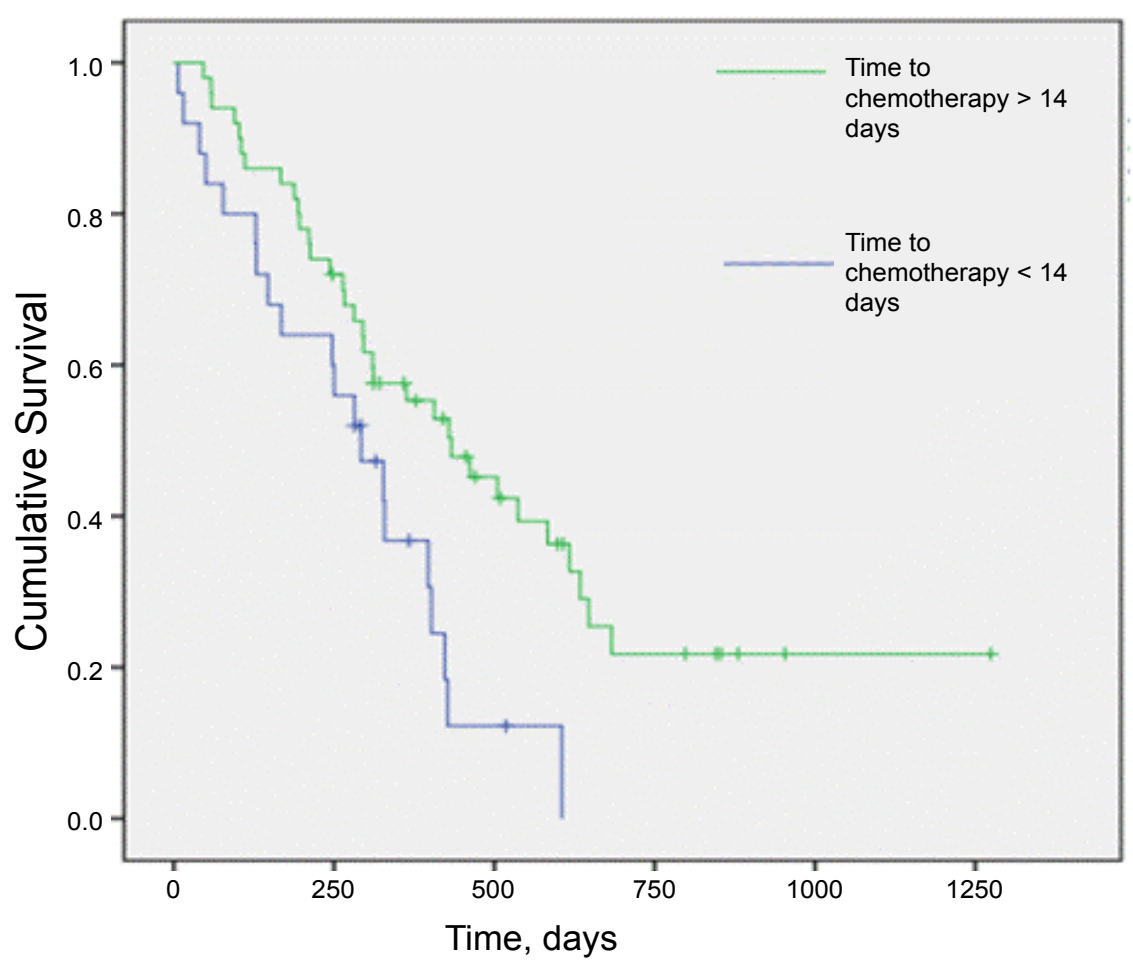

Figure 1: Overall survival for all patients stratified by start time to chemotherapy. $p=0.008$.

Table 2: Logistic Regression of Factors Affecting Overall survival.

\begin{tabular}{|l|l|l|l|}
\hline Factors & Significance & Hazard & Confidence Interval (95\%) \\
\hline Age at Diagnosis & 0.983 & 1.001 & $0.941-1.065$ \\
\hline Performance Status (KPS > 70 vs. < 70) & 0.713 & 0.716 & $0.12-4.259$ \\
\hline Greater than 3 Comorbidities & 0.698 & 1.394 & $0.26-7.488$ \\
\hline Stage (limited vs. extensive) & $\mathbf{0 . 0 3 7}$ & $\mathbf{7 . 2}$ & $\mathbf{1 . 1 2 2 - 4 6 . 1 9 7}$ \\
\hline Radiation Therapy & 0.992 & 0.994 & $0.284-3.476$ \\
\hline Earliest Symptoms Longer than 59 Days & 0.919 & 1.066 & $0.309-3.683$ \\
\hline Chemo Starting More than 14 Days after Dx & 0.831 & 1.13 & $0.367-3.48$ \\
\hline
\end{tabular}

radiation treatment, and timing of symptoms to diagnosis. In the Cox proportional model only stage was found to increase the hazard of dying (Table 2).

Similarly, we looked at logistic regression examining age at diagnosis, performance status over 70 , greater than 3 comorbidities, staging, radiation, and earliest symptoms greater than 59 days found that only stagewas significantly associated with mortality. Those with extensive disease had $30 x$ odds of dying than those with limited disease (95\% Cl 1.8-517).

\section{Discussion}

SCLC is an aggressive malignancy that often presents with metastatic disease. Median survival time with treatment ranges from 7 to 9 months. Because of the aggressive nature of the disease and the possibility for rapid growth, there is potential that a delay in initiation of systemic therapy can result in adverse outcomes. Our data did not reveal any benefit to early initiation of chemotherapy in any patient subset. Furthermore, when the overall population was examined we found a detriment to early initiation of chemotherapy (Figure 1) although this was not significant on multivariate analysis. When staging subsets (limited versus extensive) are examined separately, there is no difference in outcome for early versus late initiation of chemotherapy. We were unable to find any patient subset that seemed to benefit from early initiation of chemotherapy.

It is unclear why chemotherapy timing appears to have no effect. It is possible that while SCLC is an aggressive malignancy the tumor progression associated with delayed chemotherapy is for local disease only and not for distant spread. Our data does not show any benefit for early initiation of chemotherapy and furthermore there is a trend towards detriment (Figure 1). While it is very possible that study limitations are responsible for artificially creating what appears to be a chemotherapy detriment (see below) it is also possible that urgent start to chemotherapy truly is the cause for worse outcome. There is a high psychological stress level that can occur when patients are told of their diagnosis [3], this 
can be further exacerbated when patients are instructed that they must initiate chemotherapy urgently. Such stress may influence outcome. Hamer, et al. found that psychological distress was a predictor for cancer mortality, especially in lung cancer [4]. A second reason that urgent start to chemotherapy could be detrimental is if rapid chemotherapy start time undermines proper staging and evaluation of patients. Patients with unclear staging findings (such as a small pleural effusion, or liver lesion) may start chemotherapy at the expense of a full work-up and are thus inappropriately staged and/ or treated.

There is limited data regarding timeliness of care and lung cancer outcomes. Most of the studies conducted on the subject have focused on non-small cell lung cancer [5-8]. Their findings are consistent with those found in our study; that earlier treatment correlates with lower survival time and higher mortality rates. However, most studies, including our own, demonstrate that earlier treatment typically occurs in individuals with more advanced disease.

A more recent retrospective study examining SCLC found that patients seen in an outpatient setting took a significantly longer time to be treated than those presenting to an emergency department. Median times from diagnosis to treatment were 15 days vs. 39 days in the ED vs. the outpatient clinic respectively. The study also found that the two populations had no statistical difference in survival [9].

Our study has multiple limitations, the most major of which is the limited sample size. The population size of this study severely limits its utility in examining subgroups. Larger numbers could possibly find a subset that would benefit from early initiation of chemotherapy. However, this is unlikely given there was no trend towards better survival in any of the subsets examined. A second weakness is unmeasured variables; there are variables that may have been important that were not recorded. For example, tumor volume may be an important metric. It is possible that patients with large tumor volume do benefit from early initiation of chemotherapy. This study attempted to account for as many variables as possible but the authors acknowledge that data collection is limited. It is also possible that patients who were symptomatic from their disease started chemotherapy earlier. These patients would be expected to do worse while at the same time benefit the most from early chemotherapy initiation. Third, we used an arbitrary time of 2 weeks as a cut-off for early versus late but when time from diagnosis to chemotherapy initiation as a continuous variable was used there was a trend towards improved outcome with later chemotherapy initiation (Pearson coefficient of 0.22 with a $p$-value of 0.058 ), thus it is likely that any time cut-off would have similar result.
The best method to determine optimal treatment timing in small cell lung cancer would be a prospective study. However, it would be unlikely that such a study would be successful as patients and providers alike may be uncomfortable with an intentional delay in chemotherapy initiation. In the absence of any level one evidence, the best evidence available must suffice. This study and similar retrospective studies provide the only available data regarding the importance of chemotherapy timing in SCLC.

\section{Conclusions}

This data is important for several reasons. We were unable to find any benefit for any patient subset with urgent initiation of chemotherapy. This indicates that physicians may safely start chemotherapy at a comfortable pace. There is no need for emergent chemotherapy. A modest delay in starting chemotherapy will allow for patients and families to better absorb the diagnosis and prognosis. Delay in treatment allows patients to make preparations for treatment and consider alternative options (second opinion, best supportive care only, etc.). From the clinical perspective, non-urgent chemotherapy allows for a more thorough staging process. From a logistical standpoint, the non-urgent approach is easier on hospital resources. When urgent chemotherapy is requested, there can be an unnecessary strain on staff, room availability, and other limited resources.

In conclusion, we did not find any benefit to urgent initiation of chemotherapy in any subset of SCLC patients. Based on this data, chemotherapy can be initiated at a comfortable pace without concern for detriment.

\section{Conflicts of Interest}

None.

\section{Funding}

None.

\section{References}

1. Simon GR, Wagner $H(2003)$ Small cell lung cancer. Chest 123: $259 \mathrm{~s}-271 \mathrm{~s}$

2. vanMeerbeeck JP, Fennell DA, De Ruysscher DK (2011) Small-cell lung cancer. Lancet 378: 1741-1755.

3. Morrison EJ, Novotny PJ, Sloan JA, Yang P, Patten CA, et al. (2017) Emotional Problems, Quality of Life, and Symptom Burden in Patients With Lung Cancer. Clin Lung Cancer 18: 497-503.

4. Hamer M, Chida Y, Molloy GJ (2009) Psychological distress and cancer mortality. Journal of Psychosomatic Research 66: $255-258$.

5. Myrdal G, Lambe M, Hillerdal G, Lamberg K, Agustsson T, et al. (2004) Effect of delays on prognosis in patients with non-small cell lung cancer. Thorax 59: 45-49.

6. Gould MK, Ghaus SJ, Olsson JK, Schultz EM (2008) Timeliness of Care in Veterans With Non-small Cell Lung Cancer. Chest 133: 1167-1173. 
7. Bozcuk H, Martin C (2001) Does treatment delay affect survival in non-small cell lung cancer? A retrospective analysis from a single UK centre. Lung Cancer 34: 243-252.

8. Annakkaya AN, Arbak P, Balbay O, Bilgin C, Erbas M, et al. (2007) Effect of symptom-to-treatment interval on prognosis in lung cancer. Tumori 93: 61-67.

9. Haque N, Raza A, Mcgoey R, Boulmay B, Diethelm L, et al. (2012) Small Cell Lung Cancer: Time To Diagnosis And Treatment. Southern Medical Journal 105: 418-423. 